\title{
Efecto de la maduración en la conservación de la carne de potro
}

\author{
M. Ruiz', M.J. Beriain ${ }^{1}$, K. Insausti ${ }^{1}$, J.M. Lorenzo ${ }^{2}$, M.J. Cantalejo ${ }^{1}$ \\ y M.V. Sarriés ${ }^{1, *}$
}

1 IS-FOOD. Research Institute for Innovation \& Sustainable Development in Food Chain. Escuela Técnica Superior de Ingenieros Agrónomos, Universidad Pública de Navarra, Campus de Arrosadía, 31006 Pamplona, España

2 Centro Tecnológico de la Carne de Galicia, Rúa Galicia N 4, Parque Tecnológico de Galicia, San Cibrao das Viñas, 32900 Ourense, España

\section{Resumen}

Para el desarrollo del presente estudio, se investigó el efecto de la maduración del músculo Longissimus dorsi (LD), por un lado como pieza entera y por otro lado tras el fileteado, sobre los parámetros de calidad de la carne de potro. Se emplearon músculos madurados 24 horas en la canal procedentes de ocho potros quincenos de raza Burguete. Cada lomo se dividió en dos partes iguales. Una parte no fue madurada, y la segunda fue madurada 7 días. Posteriormente, ambas partes fueron fileteadas y los filetes fueron conservados durante 9 días en bandejas cubiertas con film permeable al oxígeno en un expositor. No hubo interacción entre la maduración en el lomo y el tiempo de conservación posterior del filete. Los valores de textura fueron bajos desde el comienzo siendo considerada "tierna". Debido a la maduración, el enrojecimiento $\left(a^{*}\right)$ fue intenso y el contenido de metamioglobina fue bajo, pero la oxidación de los lípidos y la degradación del olor aumentaron. Cuando la maduración se llevó a cabo en filete, el tiempo de conservación y la atmósfera rica en oxígeno hicieron que la carne de potro se deteriorara rápidamente. La oxidación de los lípidos y la mioglobina y la degradación del color aumentaron día a día. Además, cuanto más tiempo estuvo expuesta la carne al oxígeno, peores fueron las valoraciones de color y olor sensorial de la carne de potro. El tiempo de conservación de la carne de potro fue inferior a 3 días, siendo el color característico el factor limitante.

Palabras clave: Potro, maduración, textura, degradación de color, oxidación, análisis sensorial.

\section{Abstract \\ Ageing effect on foal meat preservation}

Ageing effect in loin and steaks was investigated to study quality parameters on foal meat. Eight Longissimus dorsi muscles (LD) aged 24 hours inside the carcass from 15-month-old foals were used. One equal part of the loin was not aged, the second part was aged 7 days and both of them were filleted in steaks, aged for 9 days in trays and preserved in an expositor chamber. There was no interaction between loin ageing time and steak conservation time. Texture values were low at the beginning, the foal meat being described as "tender". Redness ( $\left.a^{*}\right)$ was intense and metmyoglobin content was low, but lipid oxidation and odor degradation increased. When ageing was carried out on steaks, oxygen exposure time and the free oxygen atmosphere made foal meat deteriorate rapidly. Lipid and myoglobin oxidation and color degradation increased day by day. In addition, the longer the meat was exposed to free oxygen, the worse were the scores for sensory color and odor of foal meat. The preservation time of foal meat was less than 3 days, the characteristic color being the limiting factor.

Keywords: Foal, ageing, texture, color degradation, TBAR'S, sensory analysis.

* Autor para correspondencia: vsarries@unavarra.es

https://doi.org/10.12706/itea.2018.004 


\section{Introducción}

El color y la terneza son dos de las características más importantes de la carne en el momento de compra y de consumo (Koohmaraie, 1996; Rentfrow et al., 2004). La maduración es un proceso muy importante, cuyo principal objetivo es el ablandamiento de la carne. Pero, en este proceso no sólo ocurren cambios en la textura, sino que también tienen lugar alteraciones en el color, aroma y en la apariencia. Debido a la importancia de este proceso, muchos estudios han tratado de definirlo en diferentes especies; principalmente en vacuno y especialmente en diferentes razas de la misma, con el fin de conocer su punto óptimo de maduración (Campo et al., 2000). Sin embargo, este tipo de estudios no se ha extendido a razas equinas. La carne de potro destaca especialmente por su terneza a las 24 horas post mortem. Según la clasificación descrita por Belew et al. (2003) de acuerdo a la fuerza de corte (WBSF, Warner Bratzler Shear Force), la carne de potro, únicamente madurada 24 horas en la canal podría considerarse como "tierna" $(3,2<$ WBSF $<3,9 \mathrm{~kg})$ o incluso "muy tierna" (WBSF < 3,2 kg) según los valores descritos por varios autores (Sarriés y Beriain, 2006; Franco et al., 2011a; Lorenzo et al., 2013). La falta de valores de referencia para este proceso ha dado lugar al desarrollo de diferentes estudios. Sarriés y Beriain (2006) estudiaron el músculo LD proveniente de potros de raza Burguete, madurado 24 horas dentro de la canal y 4 y 8 días fuera de la misma. Lanza et al. (2009) desarrollaron su estudio en el músculo LD de potros de raza Sanfratellano y Haflinger con una maduración de 4 y 6 días dentro de la canal. Otros autores como Gómez y Lorenzo (2012), Lorenzo y Gómez (2012) y Lorenzo et al., (2013) Ilevaron a cabo su estudio de maduración con el músculo LD proveniente de potros de raza Gallego de Monte y de potros cruzados Hispano-Bretón x Gallego de Monte, madurado 24 horas dentro de la canal y tras un posterior fileteado. Sin embargo, no hay suficientes estudios sobre el sistema más apropiado de maduración y sobre el manejo de la carne durante este periodo. Además, la falta de normas y directrices a seguir durante este proceso, hace que no sea posible ofrecer al consumidor un producto bien definido.

Por tanto, el objetivo de este estudio fue determinar el efecto de la maduración sobre los parámetros de calidad de la carne de potro y saber si existen diferencias entre la maduración de toda la pieza de lomo fuera de la canal y la maduración después de filetear el lomo.

\section{Material y métodos}

\section{Material animal y diseño experimental}

Para llevar a cabo este estudio, se emplearon 8 potros de raza Burguete de 15 a 16 meses de edad. El manejo animal fue similar al indicado en previos estudios en los cuales se empleó esta misma raza (Sarriés y Beriain, 2005). Los animales fueron sacrificados en el matadero oficial "La Protectora" S.A. de Pamplona (Navarra) (Directiva del Consejo de la Unión Europea 95/221EC). El peso de las canales fue de $281 \pm 14,87 \mathrm{~kg}$. La media canal izquierda de cada animal fue conservada en refrigeración durante 24 horas a $0^{\circ} \mathrm{C}$, con una humedad relativa del $98 \%$ y con una corriente de aire de $13.600 \mathrm{~m}^{3} / \mathrm{h}$. Tras 24 horas, el músculo LD fue extraído de la media canal izquierda y llevado en refrigeración a la Universidad Pública de Navarra.

El músculo LD fue dividido en dos partes iguales, entre la $6^{\mathrm{a}}$ y la $7^{\mathrm{a}}$ costilla. La zona craneal fue destinada a estudiar la carne 24 horas post mortem (M0) y la zona lumbar fue madurada 7 días envuelta en una doble película de PVC permeable al oxígeno y conservada en refrigeración durante 7 días (M7) a $4 \pm 1{ }^{\circ} \mathrm{C}$ de temperatura, oscuridad total y 
$90-95 \%$ de humedad relativa. Transcurridos ambos tiempos de maduración (M0, M7), las muestras fueron fileteadas obteniendo 4 filetes de 1,5-2 cm de espesor de cada mitad. Dicho filetes fueron conservados durante 0 , 3, 6 y 9 días (tiempo de conservación: TC) en bandejas de polietileno de 0,75 litros (0750639015) cubiertas con una película de PVC permeable al oxígeno (transmisión de oxígeno: $\left.1762 \mathrm{~cm}^{3} / 254 \mathrm{~cm}^{2} / 24 \mathrm{~h} / \mathrm{atm}\right)$. Estas bandejas se almacenaron a $2 \pm 1{ }^{\circ} \mathrm{C}$, simulando las condiciones de venta al por menor en los supermercados en una cámara de refrigeración iluminada por una luz fluorescente blanca durante 10 h / día.

\section{Composición química}

La composición química de la carne de potro se analizó 24 horas después del sacrificio. El contenido de humedad (\%), proteína (\%), grasa intramuscular (\%) y ceniza (\%) se determinó siguiendo las Normas Internacionales ISO R-1442: 1978, ISO R-937: 1978, ISO R-1443: 1978 e ISO R-936-1998, respectivamente. Se cuantificó el contenido de hierro total (mg/ $100 \mathrm{~g}$ de carne fresca) (Norma UNE-EN-14082: 2003), de hierro hemínico ( $\mathrm{mg} / 100 \mathrm{~g}$ de carne fresca) y de mioglobina ( $\mathrm{mg} / \mathrm{g}$ de músculo) (Boccard et al., 1981). Además, se analizó el contenido de colágeno total ( $\mathrm{g} / 100 \mathrm{~g}$ de carne) (CT) y soluble (\% CT) (CS) de acuerdo con Bonnet y Kopp (1986).

\section{Textura instrumental}

Los parámetros de textura se determinaron tanto en el músculo LD no maduro (M0) como madurado 7 días (M7). El análisis de compresión se desarrolló en carne cruda utilizando un dispositivo de compresión modificado que evita el alargamiento transversal de la muestra (Lepetit y Culioli, 1994). Las variables consideradas fueron la compresión al $20 \%\left(\mathrm{~kg} / \mathrm{cm}^{2}\right)$ (C20) y compresión al
$80 \%\left(\mathrm{~kg} / \mathrm{cm}^{2}\right)$ (C80). La compresión al 20\% es una medida de la fuerza de las miofibrillas y la compresión al $80 \%$ está relacionada con el contenido de colágeno y el número de reticulaciones entre las moléculas de colágeno (Lepetit y Culioli, 1994). El análisis de fuerza al corte se realizó cocinando la carne envasada al vacío en un baño de agua a $75 \pm 1^{\circ} \mathrm{C}$ hasta una temperatura interna de $70 \pm 1^{\circ} \mathrm{C}$ durante 45 minutos. Esta temperatura fue medida con una sonda Digitron 3246 (Hereford, RU). La fuerza de cizallamiento máxima se evaluó utilizando un dispositivo de fuerza de cizalla Warner-Bratzler $(\mathrm{Kg})$ hasta que las muestras se cortaron completamente. En ambos casos, se analizaron un mínimo de ocho muestras de $1 \mathrm{~cm}^{2}$ (sección transversal cuadrada), con las fibras musculares paralelas al eje longitudinal de la muestra. Se utilizó un texturómetro TA-XT2i Stable Micro Systems conectado a un ordenador con IBM Foxen, microprocesador AutenticAMD-K6 (tm) y procesador 3D. Para el control del dispositivo y el procesamiento de los datos, se utilizó el programa informático "Texture Expert" versión 1.22 a Windows (Stable Micro Systems, Surrey, UK).

\section{Oxidación lipídica}

La oxidación lipídica se determinó en los filetes del músculo LD no madurado (M0) y madurado 7 días (M7) en los días 0 y 9 de tiempo de conservación (TC). El valor del ácido tiobarbitúrico (TBA) se calculó según lo descrito por Tarladgis et al. (1960). Los resultados se expresaron como mg de malonaldehído / kg de carne.

\section{Color instrumental}

El color de la carne se analizó en los filetes de M0 y M7 en los días 0, 3, 6 y 9 de TC. Los valores de color de CIE (1976) Luminosidad $\left(L^{*}\right)$, índice de rojo ( $\left.a^{*}\right)$ e índice de amarillo 
$\left(b^{*}\right)$ fueron medidos con un espectrofotómetro Minolta CM2002, iluminante D65 y un 10 Observador estándar. Se realizaron 5 medidas en cinco zonas diferentes de cada filete, cambiando la orientación del instrumento cada vez, y se trabajó con la media obtenida de estas 5 repeticiones. Posteriormente, la cromaticidad ( $C^{*}$ y los valores de tonalidad $\left(h^{*}\right)$ fueron calculados a partir de las siguientes ecuaciones: $C^{*}=(a * 2+b * 2)^{0.5}$ $y h^{*}=\arctan (b * / a *)$.

Por otro lado, se determinó la estabilidad del color en cada momento de TC $(0 d, 3 d$, $6 \mathrm{~d}$ y $9 \mathrm{~d}$ ) y se basó en la cuantificación de la metamioglobina sobre la superficie de la carne (MMb\%) (American Meat Science Association (AMSA), 2012). Los valores de reflectancia empleados fueron los correspondientes a los puntos isobésticos $525 \mathrm{~nm}$ y $572 \mathrm{~nm}$, característicos de la curva de metamioglobina. A partir de estas reflectancias se obtuvieron los valores $\mathrm{K} / \mathrm{S}$ de las curvas de referencia según la ecuación:

$$
k / S=\frac{(1-R)^{2}}{2 R}
$$

El porcentaje de MMb se calculó mediante la fórmula:

$\% M M b=\frac{K / S_{572} / K / S_{5250 \% M M b}-K / S_{572} / K / S_{5250 \% \text { MMb de la muestra }}}{K / S_{572} / K / S_{5250 \% M M b}-K / S_{572} / K / S_{525100 \% M M b}}$

\section{Análisis sensorial}

Estos análisis se realizaron en todos los filetes $(\mathrm{M} 0, \mathrm{M} 7)$ y en los días $0,3,6$ y 9 de TC. Los atributos estudiados fueron el olor y el color característico de la carne de potro fresca. Se empleó la metodología utilizada por Gómez et al. (2014) en vacuno, pero adaptada para carne de potro (Ruiz et al., 2016). Se llevaron a cabo cinco sesiones de entrenamiento específico de carne de potro descrito por los mismos autores. Los panelistas fueron entrenados con muestras reales y cuya descriptiva aparece en la tabla 1. Un panel sensorial de 15 miembros evaluó ambos atributos. Se utilizó una escala lineal no estructurada de $150 \mathrm{~mm}$ para medir la evolución del olor y el color característico. Se indicó: en el extremo izquierdo (valor mínimo, $0 \mathrm{~mm}$ ) "olor característico", "color característico" y en el extremo derecho (valor máximo $150 \mathrm{~mm}$ ) "olor no característico", "color no característico", respectivamente. El punto medio $(75 \mathrm{~mm})$ marcó el límite entre "característico" y "no característico" $(0<75 \leq 150$ $\mathrm{mm})$. Las sesiones se programaron en función de la maduración del músculo LD en pieza entera $(\mathrm{M} 0, \mathrm{M} 7)$ y en filetes $(0 \mathrm{~d}, 3 \mathrm{~d}, 6 \mathrm{~d}$ y 9d). El olor fue el primer atributo evaluado con luz roja (100 lux) seguido del color, utilizando, en este caso, luz blanca (450 lux). Las muestras fueron identificadas por un número de tres dígitos en un orden aleatorio. La cata se realizó en la sala de análisis sensorial de la Universidad Pública de Navarra acreditada según la norma UNE EN ISO / IEC 17025 (Acreditación 300 / LE 584, 2001).

\section{Análisis estadístico}

Todos los análisis estadísticos se realizaron utilizando el paquete informático SPSS 22.0. Se realizaron análisis descriptivos estadísticos para caracterizar la carne de potro en su origen y se calcularon coeficientes de variación para explicar la heterogeneidad de este tipo de carne.

$$
Y_{i j k}=\mu+\varepsilon_{i j k}
$$

siendo: $Y_{\text {ijk }}$ : La observación del parámetro analizado; $\mu$ : Media mínima cuadrada de la población; $\varepsilon_{\mathrm{ijk}}$ : Error vinculado a cada observación.

Además, para las variables en las que se estudió el efecto de la maduración del músculo LD en pieza entera y del tiempo de conservación en filetes, se realizó un análisis de varianza (ANOVA):

$$
Y_{i j k}=\mu+P_{i}+F_{j}+L_{i} x F_{j}+\varepsilon_{i j}
$$




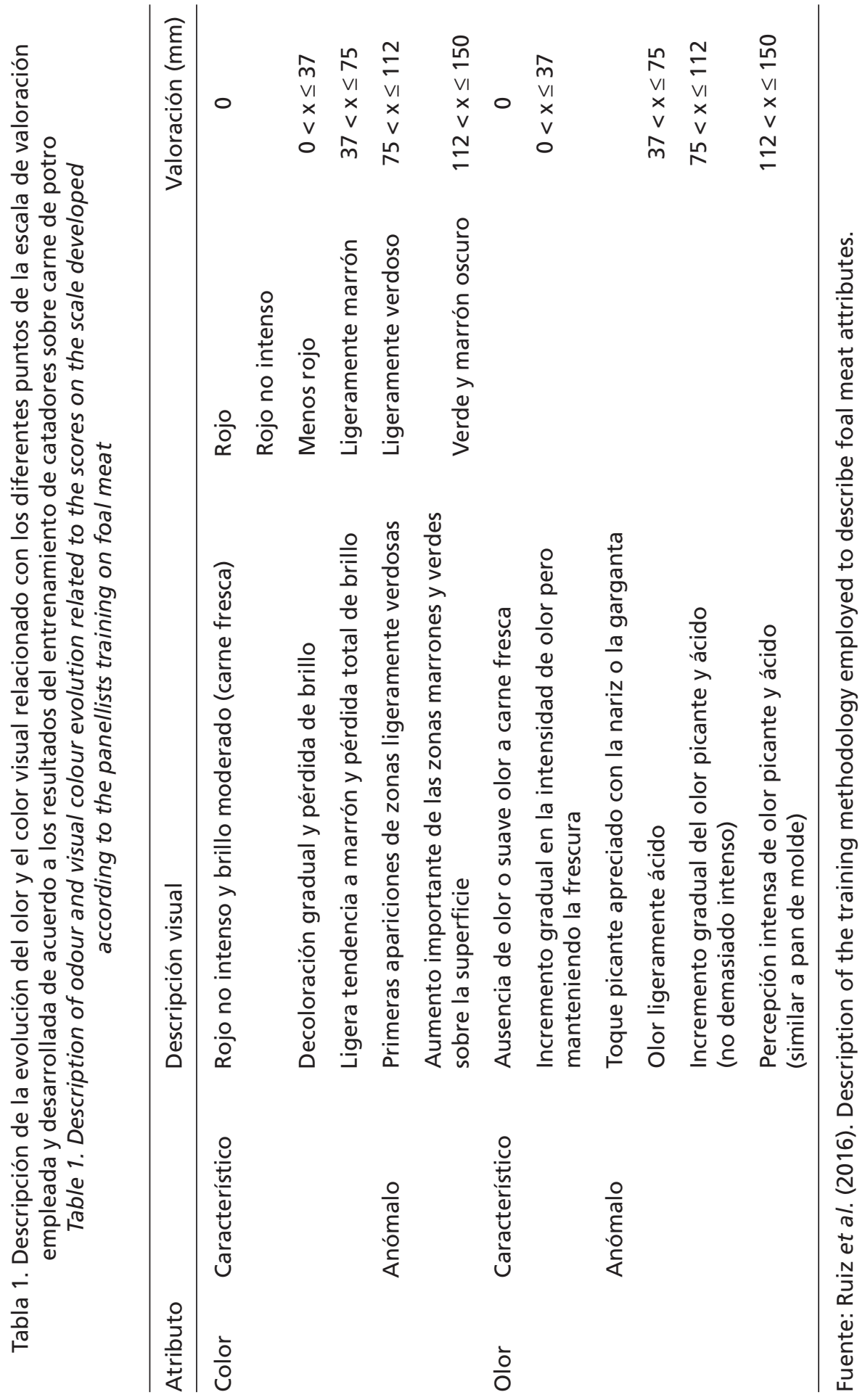


siendo: $Y_{\mathrm{ijk}}$ : La observación del parámetro analizado; $\mu$ : Media mínima cuadrada de la población; $P_{\mathrm{i}}$ : Efecto de la maduración en pieza entera. $\mathrm{i}=1$ : 0 días; $\mathrm{i}=2$ : 7 días; $\mathrm{F}_{\mathrm{j}}$ : Efecto del tiempo de conservación en filete. $j=1: 0$ días; j = 2: 3 días; j = 3: 6 días; j = 4: 9 días; PxF: Efecto de la interacción de los efectos principales; $\varepsilon_{\mathrm{ij}}$ : Error vinculado a cada observación. Las diferencias significativas $(P>0,05)$ para el tiempo de conservación fueron comprobadas con el Test de Tukey para un nivel de significación de $\mathrm{P}>0,05$.

Asimismo, se utilizaron correlaciones de Pearson para conocer la relación entre el conjunto de variables.

\section{Resultados y discusión}

\section{Composición química}

La composición química del músculo LD se muestra en la tabla 2. En general, los valores medios del contenido de humedad, cenizas y proteína se encuentran dentro del rango de valores descritos previamente por otros autores como Lorenzo et al. (2013) en potros de raza Gallego de Monte sacrificados con 15 meses de edad, Polidori et al. (2015) en potros Martina Franca sacrificados con 12 meses de edad, o Sarriés y Beriain (2005) en potros de raza Burguete de 16 meses de edad. Destaca el contenido de grasa intramuscular, el cual se aproximó al descrito por Sarriés y Beriain (2005) $(3,17 \%)$ y sin embargo, fue muy superior al obtenido por otros autores (Lorenzo et al., 2013; Polidori et al., 2015) (0,22 \% en Gallego de Monte; 1,87 \% en Martina Franca, respectivamente). Estas diferencias en grasa intramuscular podrían deberse a la raza (Lorenzo et al., 2014). Por otro lado, también es destacable el contenido de mioglobina $(\mathrm{mg} / \mathrm{g}$ músculo). Dichos valores fueron muy superiores a los obtenidos por Tateo et al. (2008)
$(3,20)$ y Sarriés y Beriain $(2005)(3,51)$. Según Lorenzo et al. (2014) un sistema de producción extensivo ayuda a incrementar el contenido de hierro de la carne de potro. Los animales a estudio permanecieron libres hasta el día previo al sacrificio. Además, su cría en alta montaña (800 msnm), ejercicio y pastoreo, exigen al organismo una oxigenación más alta y dificultosa y por lo tanto da lugar a una mayor cantidad de pigmentos en el músculo (Sañudo, 1993).

Tabla 2. Composición química del músculo Longissimus dorsi procedente de potros de raza Burguete

Table 2. Chemical composition of Longissimus dorsi muscle from Burguete foals

\begin{tabular}{lr}
\hline & \multicolumn{1}{c}{ Valores* } \\
\hline Humedad (\%) & $71,40 \pm 0,81$ \\
Ceniza (\%) & $1,14 \pm 0,03$ \\
Proteína (\%) & $23,46 \pm 0,91$ \\
Grasa intramuscular (\%) & $5,13 \pm 1,15$ \\
Hierro hemínico (mg /100 g carne) & $1,92 \pm 0,31$ \\
Mioglobina (mg/ g músculo) & $5,67 \pm 0,93$ \\
Hierro total (mg/ 100 g carne) & $2,00 \pm 0,19$ \\
\hline
\end{tabular}

*Valores indicados como media \pm desviación estándar $(n=8)$.

\section{Efecto de la maduración en la carne de potro}

El efecto de la maduración en el músculo LD en pieza entera (P) y el tiempo de conservación en los filetes ( $F$ ) no mostró ninguna interacción (PxF) (tabla 3). La maduración del músculo LD (P) sólo presentó diferencias significativas en el contenido de colágeno total $(P \leq 0,01)$. Por el contrario, el tiempo de conservación en los filetes (F) mostró un efecto más evidente en la mayo- 


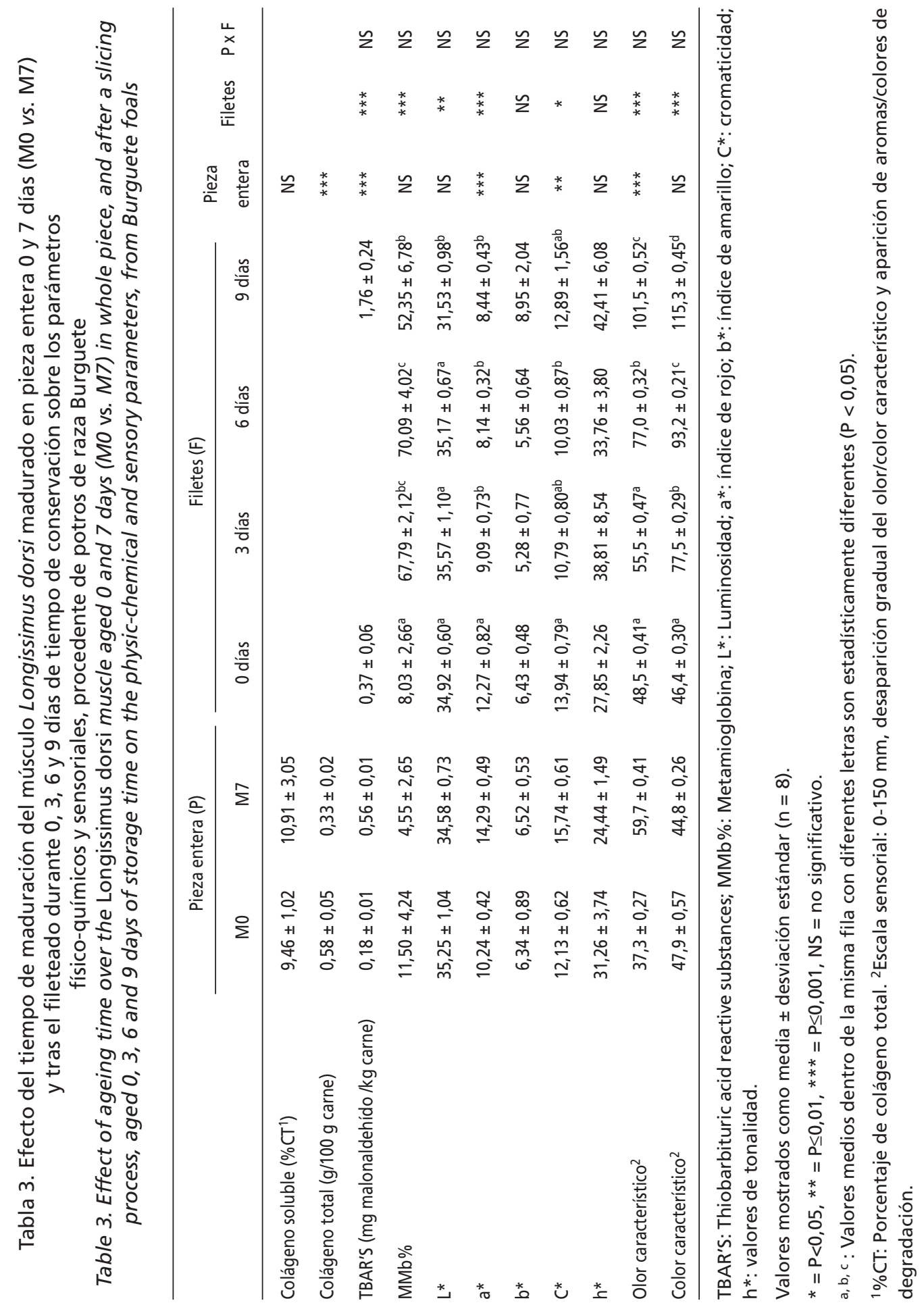


ría de las variables asociadas con los procesos de oxidación y degradación. Este resultado deja ver que la evolución de los parámetros durante el tiempo de conservación seguiría una misma tendencia independientemente del tiempo de maduración del lomo.

\section{Efecto de la maduración del músculo LD en pieza entera}

El efecto de la maduración en pieza entera se muestra en la tabla 3. Por un lado, el contenido de colágeno total difiere $(P \leq 0,001)$ entre el músculo no madurado (M0) y madurado 7 días (M7). Sin embargo, la maduración no influyó en el contenido de colágeno soluble $(P>0,05)$, ya que se obtuvieron resultados similares. Los resultados de colágeno total mostrados por Badiani et al. (1997) se encontraban entre $0,98 \%$ y $1,53 \%$ en carne madurada 10 y 14 días y procedente de potros sacrificados con 6 y 10 meses de edad; valores muy superiores a los obtenidos en este estudio. Por otro lado, los resultados de colágeno soluble en el LD no maduro (M0) fueron similares a los descritos por Sarriés y Beriain (2005) $(10,23 \pm 2,06)$. Es importante indicar que el colágeno es una estructura muy compleja, cuyo estado depende de sus características bioquímicas, polimerización y grado de reticulación, y la naturaleza, tipo y número de uniones (Lepetit y Culioli, 1994). Durante la maduración, se produjo una disminución significativa del contenido total de colágeno, que varió de 0,58 a 0,33 (tabla 3). Esto implicaría un cambio en la disposición de las fibras y en los enlaces que mantienen la estructura de colágeno. Roncalés (2001) indicó que en la carne de vacuno, la maduración no influye sobre el tejido conectivo; y Lepetit (2007) sostuvo que el colágeno en carne de vacuno se degrada después de un proceso de maduración volviéndose más soluble. Por el contrario, en nuestro estudio, la degradación del colágeno en carne de potro duran- te la maduración podría ser debida a cambios en la fracción insoluble de colágeno. Esos cambios pueden ser causados por factores desconocidos que actúan de manera diferente a los de la carne de vacuno.

En cuanto a los valores de compresión, el músculo no madurado (M0) y madurado 7 días (M7) mostraron diferencias por efecto de la maduración en la compresión al $20 \%$ o al $80 \%$ con un nivel de significación de $\mathrm{P}<0,1$. La diferente tendencia encontrada entre M0 y M7 aparece representada en las figuras A.1. y A.2. La compresión al $20 \%$ está relacionada con la disposición y la fuerza de las miofibras musculares (actina y miosina) (Lepetit y Culioli, 1994). Sin embargo, en el presente estudio, las calpainas y las catepsinas podrían no haber mostrado actividad efectiva durante la maduración y no haber deteriorado las miofibrillas; o quizás, puesto que la carne se considera tierna, ya habrían actuado lo suficiente tras $24 \mathrm{~h}$. Sin embargo, estudios desarrollados en la carne de vacuno (Koohmaraie, 1996) sostienen que la actina y la miosina no se degradan hasta los 14 ó 18 días de maduración, ya que estas proteínas son resistentes a la proteólisis causada por calpainas. Con respecto a la compresión al $80 \%$, los valores obtenidos $(2,15$ $\pm 0,67$, para $\mathrm{M0} ; 2,86 \pm 1,46$, para M7) fueron inferiores a los descritos por Sarriés et al. (2006) en carne de potro madurada 4 días $(3,52 \pm 1,49)$. La compresión al $80 \%$ está relacionada con el contenido de colágeno y sus propiedades (Lepetit y Culioli, 1994). En este caso, como la fracción soluble no varía con la maduración, tal vez sea la fracción insoluble la implicada en los cambios de compresión obtenidos.

Hubo un efecto significativo de la maduración en los resultados obtenidos de WBSF con un nivel de $P<0,1$, y destacan los bajos valores en el músculo LD madurado 7 días (Figura A.2). Estos resultados coinciden con los indicados por Sarriés y Beriain (2006) en 


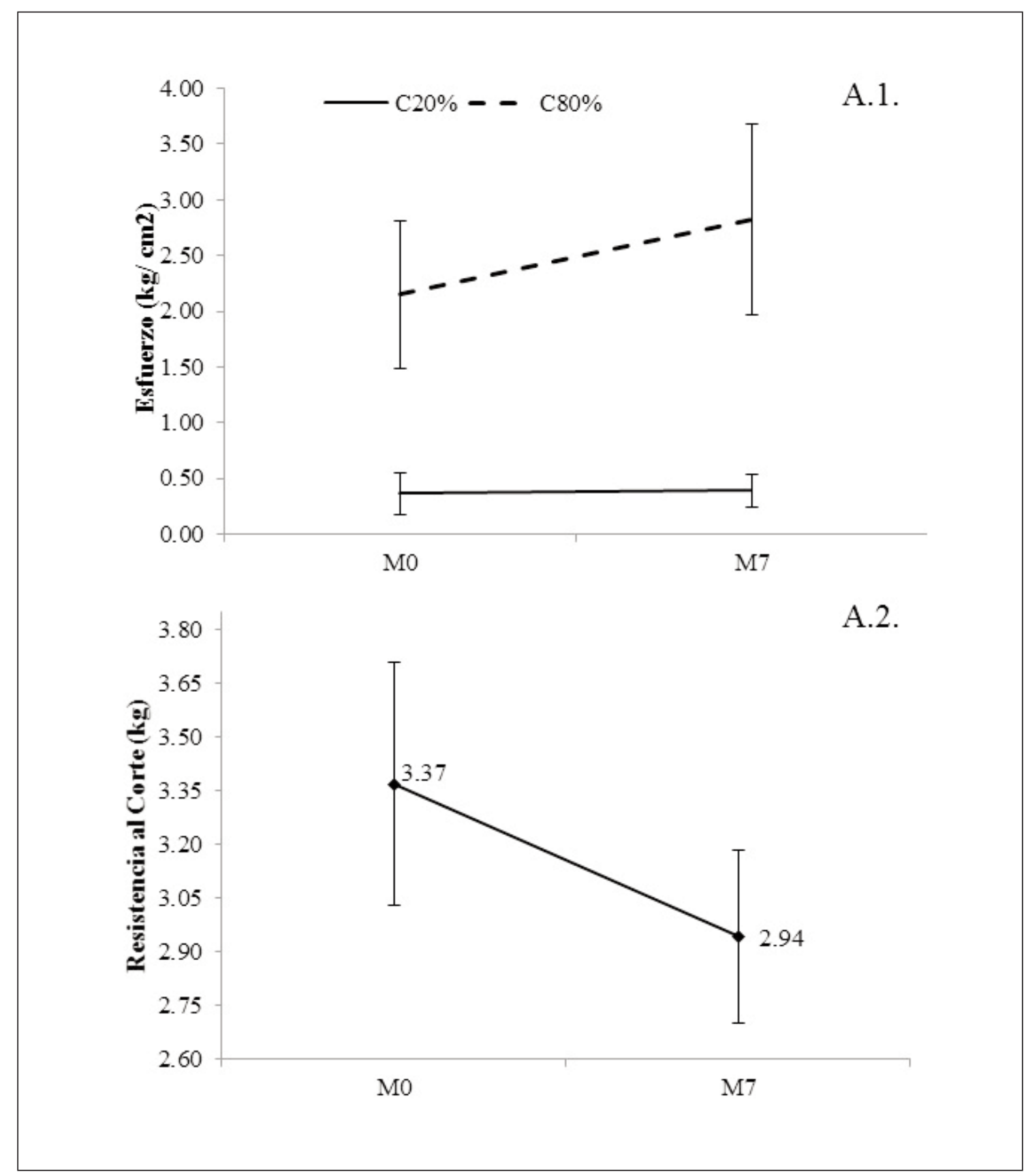

Figura A. Efecto de la maduración en la compresión (C) (A.1) y el la resistencia al corte (A.2) de muestras de carne de potro procedente del músculo Longissimus dorsi sin madurar (M0) y madurada 7 días en pieza entera (M7).

Figure A. Ageing effect at compression (C) (A.1) and shear force (A.2) of foal meat samples from the non-aged Longissimus dorsi (MO) and aged 7 days (M7). 


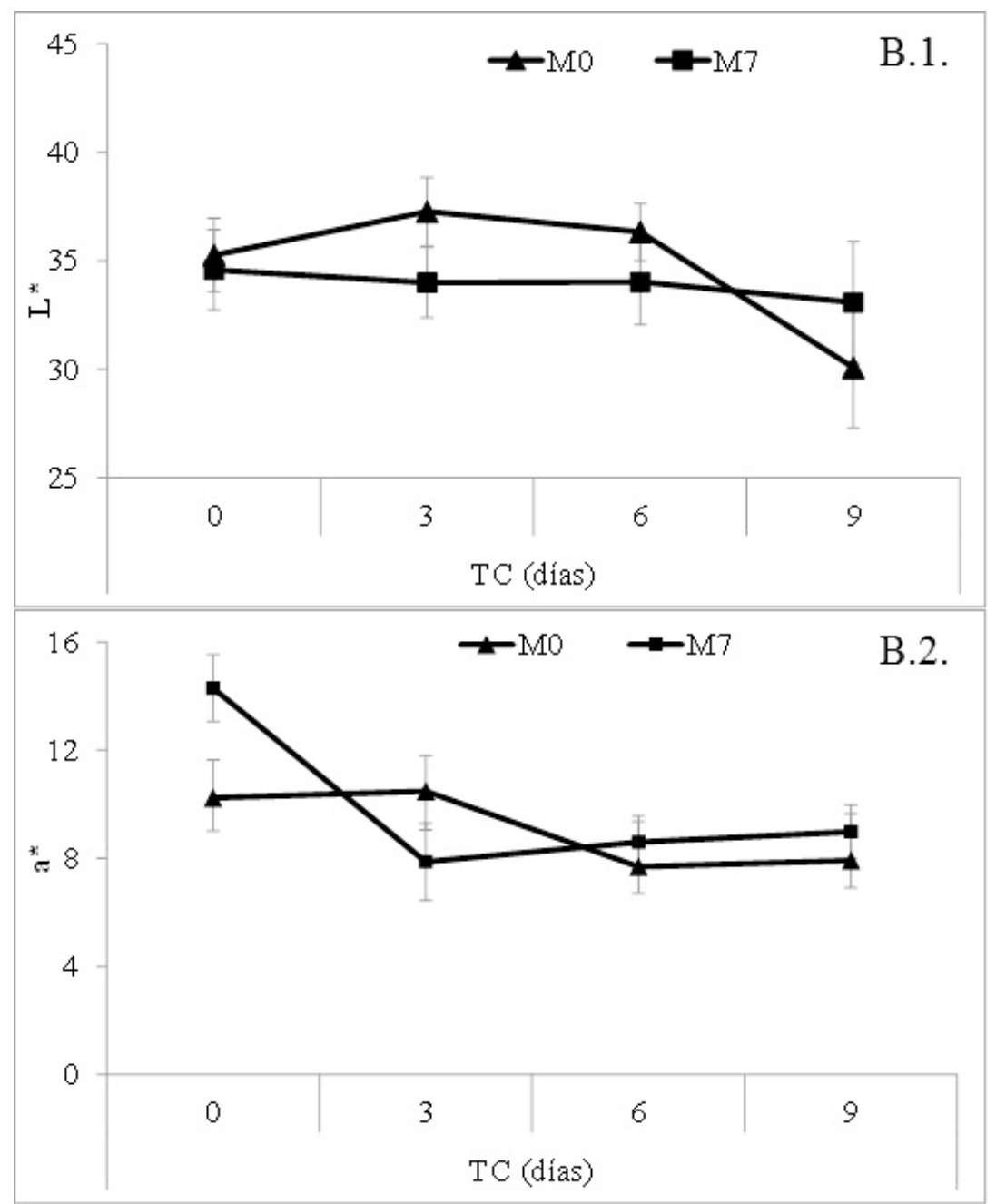

Figura B. Evolución de la luminosidad ( $\left.L^{*}\right)$ (B.1) e índice de rojo (a*) (B.2) en carne de potro fileteada y conservada durante $0,3,6$ y 9 días, procedente de lomo Longissimus dorsi no madurado (M0) y madurado 7 días (M7).

Figure $B$. Brightness $\left(L^{*}\right)$ and redness ( $\left.a^{*}\right)$ evolution in foal meat steaks during 0, 3, 6 and 9 days from the non-aged Longissimus dorsi (MO) and aged 7 days (M7).

carne de potro madurada $4(4,89 \pm 0,88)$ y 8 días $(3,59 \pm 1,18)$, pero fue ligeramente inferior a los descritos por Tateo et al. (2008) $(5,61)$ y Lorenzo et al. (2013) $(3,49 \pm 1,86)$. Bertola et al. (1994) sostienen que la terneza de la carne varía en el proceso de cocción, siendo el calor la razón de los cambios en la estructura del colágeno y de las proteínas miofibrilares. De acuerdo con la clasificación de la terneza y a las categorías establecidas por Belew et al. (2003), la carne podría considerarse entre "tierna" $(3,2 \mathrm{~kg}<$ WBSF $<3,9 \mathrm{~kg}$ ) (M0) y "muy tierna" (WBSF < $3,2 \mathrm{~kg}$ ) (M7). Según los resultados de textu- 
ra obtenidos, la carne de potro utilizada en este estudio ya fue tierna tras solamente 24 horas post mortem. Este hecho es de gran importancia a la hora de establecer tiempos de maduración para este tipo de carne.

Con respecto a la oxidación lipídica, la maduración mostró un efecto significativo $(\mathrm{P} \leq$ 0,001 ) (tabla 3). Los resultados indicaron un aumento evidente de la oxidación lipídica de 0 a 7 días de maduración. La carne de potro es rica en ácidos grasos poliinsaturados, que son altamente propensos a la oxidación (Tateo et al., 2008; Lorenzo y Gómez, 2012). Por lo tanto, el tiempo de maduración podría ser una causa directa de la oxidación de lípidos.

La oxidación de la mioglobina está representada por el contenido de $\mathrm{MMb}(\mathrm{MMb} \%)$ sobre la superficie de la carne (tabla 3). La maduración no mostró influencia sobre la misma $(P \geq 0,05)$; aunque puede ser debido a la elevada variabilidad mostrada entre animales (mayor del 30\%). Sin embargo, se puede apreciar una clara diferencia en el contenido entre ambos tiempos de maduración $(11,50 \pm 4,24$ en $\mathrm{M0}$ vs. 4,55 $\pm 2,65$ en M7) (\%). Este hecho podría explicar que un proceso de maduración mantiene la mioglobina en su estado oxigenado durante más tiempo (oximioglobina). Sarriés y Beriain (2006) afirmaron que con un proceso de maduración hay una reducción en la tasa de consumo de oxígeno en la carne; lo que provoca un aumento de la capa de oximioglobina, responsable del color rojo de la carne y su intensidad.

Los valores de las coordenadas de color aparecen en la tabla 3. Al contrario de la mayoría de los parámetros estudiados, hubo una variación muy baja entre muestras para $L^{*}$, $a^{*}$ y $C^{*}(<5 \%)$, pero alta en los parámetros $b^{*} y h^{*}(20-30 \%)$. La maduración no mostró ningún efecto sobre $L^{*}, b^{*}$ y $h *(P>$ $0,05)$, pero sí sobre $a^{*}$ y $C^{*}(P \leq 0,001)$. El índice de rojo $\left(a^{*}\right)$ y la cromaticidad $\left(C^{*}\right)$ aumentaron con los días de maduración. Con respecto a la luminosidad $\left(L^{*}\right)$, algunos autores indican valores superiores (Gómez y Lorenzo, 2012; Lorenzo y Gómez, 2012), inferiores (Bingol y Ergun, 2011; Polidori et al., 2015) y similares (Sarriés y Beriain, 2006) a los indicados en la tabla 3. Según a* y $C^{*}$, la variación entre ambos tiempos de maduración (M0 vs. M7) fue de 28,34\% y 29,76\%, respectivamente. Esta variación podría explicar el aumento y la intensidad del color rojo en la carne de potro como consecuencia del proceso de maduración. Este hecho ya ha sido explicado por Sarriés y Beriain (2006) siendo atribuido a la baja tasa de respiración mitocondrial como resultado del almacenamiento refrigerado. La reducción en el consumo de oxígeno hace que el espesor de la capa de oximioglobina aumente (que sea más profundo), y que el color rojo de la carne mejore; pero, en contrapartida, este color rojo se caracteriza por su baja estabilidad. Los valores de índice de rojo $\left(a^{*}\right)$ en la carne de potro descritos por otros autores (Lanza et al., 2009; Bingol y Ergun, 2011; Lorenzo y Gómez, 2012) fueron similares a los obtenidos en el presente estudio para el músculo LD madurado 7 días (M7). Sin embargo, los resultados obtenidos por Polidori et al. (2015) fueron similares a los del músculo no madurado (M0). Por el contrario, estos resultados fueron muy inferiores a los descritos por King et al. (2012) (a*: 32-15) o Kurvea et al. (2016) $\left(a^{*}: 28,5\right)$ en carne de vacuno.

Los valores de olor y color característico aparecen en la tabla 3. La maduración no mostró ningún efecto sobre el color $(P>0,05)$, pero sí sobre el olor $(P \leq 0,001)$. Parece ser que la maduración fue importante en la evaluación de este segundo parámetro. Los panelistas describieron que la carne de potro procedente del músculo no madurado (M0) era "carne sin olor". Sin embargo, no describieron lo mismo en la carne madurada 7 días quedando reflejado en una valoración más elevada. En este caso, el olor continuaba siendo a carne fresca, pero lo 
percibían de forma más intensa. Aunque no excedió el límite $(75 \mathrm{~mm})$, sí se produjo una visible degradación del olor característico. Esto puede estar relacionado con la oxidación lipídica sufrida durante la maduración, ya que mostraron una correlación positiva ( $p \leq 0,001 ; r=0,83)$. A pesar de la falta de estudios sobre el proceso de maduración en la carne de potro y su influencia sobre la evaluación sensorial de la misma, Lorenzo y Gómez (2012) mostraron resultados similares en carne de potro conservada entre 7 y 10 días sin sufrir ningún proceso de maduración previo y describieron la carne como "moderadamente aceptable". A pesar de que la maduración sí mostró un efecto sobre el color medido de forma instrumental $(P \leq 0,001)$ (tabla 3), el panel entrenado no apreció ninguna diferencia visual entre ambos tipos de carne para el "color característico" entre la carne procedente de M0 y M7, y describiendo su color como "ligeramente marrón y poco brillante".

\section{Efecto del Tiempo de conservación (TC) en filetes}

Los resultados obtenidos para los parámetros de calidad de la carne durante los días $0,3,6$ y 9 de TC se muestran en la tabla 3. La conservación en filetes (TC) mostró un efecto significativo en la oxidación lipídica ( $\mathrm{P} \leq$ $0,001)$. Esto implicó que los niveles de rancidez aumentaron con la presencia de oxígeno y el tiempo de exposición. Estos resultados coinciden con los descritos por otros autores (Gómez y Lorenzo, 2012; Zakrys et al., 2008). Si se extrapola a carne de potro, el valor máximo de oxidación no superó el umbral de rancidez descrito por Campo et al. (2006) en carne de vacuno $(2,28 \mathrm{mg}$ MDA/ kg carne fresca). Así mismo, Gómez y Lorenzo (2012) observaron que después de 10 días, la carne de potro no alcanzaba el límite mencionado.
El efecto de TC fue significativo $(P \leq 0,001)$ sobre el contenido de metamioglobina. Este parámetro aumentó de forma considerable entre el día 0 y 3 de TC $(88,02 \%)$. Esta variación podría ser la causa del desarrollo de tonos marrones o verdosos en la carne $y$, por tanto, de la disminución de la estabilidad del color (Sarriés y Beriain, 2006). Además, se describieron correlaciones negativas de $\mathrm{MMb} \%$ con $a *(P \leq 0,01 ; r=-0,72)$ y positivas de $\mathrm{MMb} \%$ con el color característico $(\mathrm{P}$ $\leq 0,01 ; r=0,59)$. Esto coincide con Zakrys et al. (2008) quienes asociaron la presencia de oxígeno con un aumento de oxidación de la oximioglobina y degradación del color.

De acuerdo con las coordenadas de color, el TC mostró un efecto significativo en la luminosidad $(P \leq 0,01)$, índice de rojo $(P \leq 0,001)$ y cromaticidad $(P \leq 0,05)$, pero no en el índice de amarillo y tonalidad $(P \geq 0,05)$ (tabla $3)$. La luminosidad ( $\left.L^{*}\right)$ se mantuvo constante durante los 6 primeros días $(35,22)$ y en ese momento, este valor disminuyó $(31,53)$ y la carne se volvió más oscura. Sin embargo, Zakrys et al. (2008) observaron el efecto contrario en muestras de vacuno debido al oxígeno libre. Es probable que este oscurecimiento se produjese debido al alto contenido de hierro hemínico que provoca una baja reflectancia de la luz (Seydim et al., 2006). Con respecto al índice de rojo (a*), su valor disminuyó día a día $(12,27$ el día 0 ; 9,09 el día 3; 8,14 el día 6; 8,44 el día 9). Otros autores (Bingol y Ergun, 2011; Gómez y Lorenzo, 2012; Leygonie et al., 2011; Lorenzo y Gómez, 2012) describieron valores similares con muestras de carne en diferentes condiciones de envasado. También se puede apreciar una relación inversa entre los valores de TBA y la estabilidad del color (valores $\left.a^{*}\right)(P \leq 0,01 ; r=0,62)$. Esta relación también se corroboró en otros estudios con el empleo de envases enriquecidos en oxígeno (Gómez y Lorenzo, 2012; Kim et al., 2010; Lorenzo y Gómez, 2012). Estos resul- 
tados indican que el oxígeno libre provocó la disminución de la estabilidad del color y el aumento de la oxidación lipídica (Zakrys et al., 2008). Es más, Spanier (1992) afirmó que la cantidad de hierro, el cual actúa como catalizador (proveniente de la hemoglobina, la mioglobina y el citocromo) y la cantidad de hierro hemínico y no hemínico está relacionada con el proceso de oxidación lipídica. Esto podría explicarse por el alto contenido de mioglobina (5,84 $\mathrm{mg} / \mathrm{g}$ de músculo) y hierro hemínico (1,97 mg / $100 \mathrm{~g}$ de carne) de la carne de potro, en este estudio.

Además, se encontraron diferencias con un nivel de significación de $\mathrm{P}<0,1$ entre $\mathrm{M} 0 \mathrm{y}$ M7 durante el TC. La evolución de ambos tipos de carne mostró una tendencia diferente en cuanto a la luminosidad (Figura B.1.) y el índice de rojo (Figura B.2.). Por un lado, los valores de $L^{*}$ en la carne procedente de $\mathrm{M0}$, se mantuvieron constantes hasta el día $6(36,28)$ y luego disminuyeron a 30,08 el día 9 , tornando más oscuras. Por otra parte, el TC no afectó a la evolución de $L^{*}$ en la carne procedente de M7, manteniendo un valor constante de 33,92. Las muestras de músculo no madurado (M0) sufrieron una variación de $26,62 \%$ entre los días 3 y 6 (10,48 vs. 7,69). Por el contrario, la variación en las muestras del músculo madurado (M7) fue más evidente $(45 \%)$ y ocurrió antes, entre los días 0 y $3(10,48$ vs. $7,69)$. Esto significa que el color de la carne madurada durante 7 días tuvo una estabilidad más baja que el color de las muestras que no se maduraron (M0). Esta disminución en el índice de rojo podría estar influenciada por la oxidación de ácidos grasos poliinsaturados propios de la carne de potro, y por el elevado incremento de metamioglobina sufrido durante los 3 primeros días. El índice de amarillo ( $b^{*}$ ) está relacionado con el color de con la grasa (Franco et al., 2011b), pero no contribuye significativamente a la apariencia de la carne (Leygonie et al., 2011). Los valores obtenidos fueron inferiores a los descritos en otros estudios (Sarriés y Beriain, 2006; Lanza et al., 2009; Gómez y Lorenzo, 2012; Lorenzo y Gómez, 2012) y ligeramente superiores a los citados por Franco et al. (2011a) en carne de potro. Los valores de cromaticidad ( $C^{*}$ ) fueron similares a los descritos en carne de potro por Franco et al. (2011b) y más bajos que los indicados por Lanza et al. (2009). Por otro lado, sólo los valores de tonalidad $\left(h^{*}\right)$ obtenidos en el día 0 de TC fueron similares a los observados en otros estudios (Franco et al., 2011b; Lanza et al., 2009), ya que tras este día (3, 6 y 9 días de TC) los valores fueron moderadamente superiores.

El tiempo de conservación (TC) presentó un efecto importante sobre el olor y el color característico de la carne de potro ( $P \leq$ 0,001 ) (tabla 3). Dicho efecto destacó claramente en la valoración del olor entre 3 y 6 días de TC ( 55 vs. $77 \mathrm{~mm}$ ). El tercer día de conservación mantenía un olor intenso pero característico. El día 6 , se superó el límite establecido $(75 \mathrm{~mm})$ percibiendo ligeras sensaciones a picor y acidez. Este punto marca la diferencia entre carne con olor característico y carne con olor que ha dejado de ser característico y se ha degradado. Los ácidos grasos poliinsaturados están muy presentes en la carne de potro y son muy susceptibles a la oxidación (Badiani et al., 1997; Sarriés y Beriain, 2006; Tateo et al., 2008), siendo este factor una de las causas principales de la degradación del olor y la consiguiente aparición de olores a moho y putrefacción apoyada por la actividad microbiana (Gómez y Lorenzo, 2012). De acuerdo al color característico, el límite fue superado tras 3 días de TC (77,5 mm); momento en el que comenzaron a aparecer las primeras tonalidades verdosas. Por lo tanto, la degradación del color fue muy precoz. Lorenzo et al. (2012) y Gómez y Lorenzo (2012) describieron un tiempo de conservación 
para carne de potro más largo ya que mantuvieron su color característico hasta 7 días y fue el atributo determinante. Cabe destacar las correlaciones encontradas entre la valoración de color y el $\mathrm{MMb} \%, \mathrm{y}$ a* $(\mathrm{P} \leq$ 0,$001 ; r=0,58, r=-0,66$, respectivamente) y $L^{*}(P \leq 0,01 ; r=-0,42)$. Esto se tradujo en la degradación del color característico como resultado de una alta oxidación y disminución del índice de rojo y de la luminosidad. En el presente estudio, el atributo sensorial que marcó el máximo tiempo de conservación de la carne de potro fue el color, ya que su degradación fue previa a la del olor e inferior a 3 días.

\section{Conclusiones}

En lo que a carne de potro se refiere, un proceso de maduración del músculo LD fuera de la canal puede no ser necesario, ya que a diferencia de otras especies como el vacuno los valores de terneza 24 horas post mortem son adecuados. Los valores de los parámetros de oxidación y color limitan la estabilidad de la carne de potro a menos de 3 días y está directamente afectada por el tiempo de maduración. Aunque son necesarios más estudios acerca de la maduración y conservación de la carne de potro, se podría conseguir un tiempo de conservación más largo de carne de potro fileteada, siempre que el oxígeno sea controlado mediante el empleo de técnicas de envasado al vacío o atmósfera controlada/modificada.

\section{Agradecimientos}

Los autores agradecen a ASCANA (Asociación Ganadera de Caballos de raza Burguete en Navarra) el apoyo y a Luis Goñi por los servicios prestados.

\section{Referencias}

AMSA (2012). Meat color measurement guidelines. Ed. American Meat Science Association. Illinois, EE.UU. 136 pp.

Badiani A, Nanni N, Gatta PP, Tolomelli B, Manfredini M (1997). Nutrient Profile of Horsemeat. Journal of Food Composition and Analysis 10: 254-269.

Belew JB, Brooks JC, McKenna DR, Savell JW (2003). Warner-Bratzler shear evaluations of 40 bovine muscles. Meat Science 64 (4): 507-12.

Bertola NC, Bevilacqua AE, Zaritzky NE (1994). Heat treatment effect on texture changes and thermal denaturation of proteins in beef muscle. Journal of Food Processing and Preservation 18 (1): 31-46.

Bingol EB, Ergun O (2011). Effects of modified atmosphere packaging (MAP) on the microbiological quality and shelf life of ostrich meat. Meat Science 88 (4): 774-785.

Boccard R, Buchter L, Casteels E, Cosentino E, Dransfield E, Hood DE, Joseph RL, MacDougall DB, Rhodes DN, Schön I, Tinbergen BJ, TouraiIle C (1981). Procedures for measuring meat quality characteristics in beef production experiments. Report of a working group in the commission of the European communities (CEC) beef production research program. Livestock Production Science 8 (5): 385-397.

Bonnet M, Kopp J (1986). Dosage du collagène dans les tissues conjonctifs de la vie et les produits carnés. Viandes Produits Carnés 7 (6): 263-266.

Campo MM, Santolaria P, Sañudo C, Lepetit J, Olleta JL, Panea B, Albertí P (2000). Assessment of breed type and ageing time effects on beef meat quality using two different texture devices. Meat Science 55 (4): 371-378.

Campo MM, Nute GR, Hughes SI, Enser M, Wood JD, Richardson RI (2006). Flavour perception of oxidation in beef. Meat Science 72 (2): 303-311.

Franco D, Fernández M, Rodríguez E, García L, Lorenzo JM (2011a). Calidad de la carne de potro Gallego de Monte en diferentes explotaciones. Archivos de Zootecnia 60: 389-392. 
Franco D, Rodríguez E, Purriños L, Crecente $S$, Bermúdez R, Lorenzo JM (2011b). Meat quality of "Galician Mountain" foals breed. Effect of sex, slaughter age and livestock production system. Meat Science 88: 292-298.

Gómez I, Beriain MJ, Sarriés MV, Insausti K, Mendizábal JA (2014). Low-fat beef patties with augmented Omega-3 fatty acid and CLA levels and influence of grape seed extract. Journal of Food Science 79 (11): S2368-S2376.

Gómez M, Lorenzo JM (2012). Effect of packaging conditions on shelf life of fresh foal meat. Meat Science 91 (4): 513-520.

Kim YH, Huff-Lonergan E, Sebranek JG, Lonergan SM (2010). High-oxygen modified atmosphere packaging system induces lipid and myoglobin oxidation and protein polymerization. Meat Science 85 (4): 759-767.

King DA, Shackelford SD, Kalchayanand N, Wheeler TL (2012). Sampling and aging effects on beef Longissimus color stability measurements. Journal of Animal Science 90: 3596-3605.

Koohmaraie M (1996). Biochemical factors regulating the toughening and tenderization processes of meat. Meat Science 43: 193-201.

Kurve VP, Joseph P, Williams JB, Kim TJ, Boland H, Smith T, Schilling MW (2016). The effect of feeding native warm season grasses in the stocker phase on the carcass quality, meat quality, and sensory attributes of beef loin steaks from grain-finished steers. Meat Science 112: 31-38.

Lanza M, Landi C, Scerra M, Galofaro V, Pennisi P (2009). Meat quality and intramuscular fatty acid composition of Sanfratellano y Haflinger foals. Meat Science 81: 142-147.

Lepetit J, Culioli J (1994). Mechanical properties of meat. Meat Science 36 (1-2): 203-237.

Lepetit J (2007). A theoretical approach of the relationships between collagen content, collagen cross-links and meat tenderness. Meat Science 76 (1): 147-159.

Leygonie C, Britz TJ, Hoffman LC (2011), Oxidative stability of previously frozen ostrich Muscular isilio fibularis packaged under different modified atmospheric conditions. International Journal of Food Science and Technology 46: 1171-1178.
Lorenzo JM, Gómez M (2012). Shelf life of fresh foal meat under MAP, overwrap and vacuum packaging conditions. Meat Science 92 (4): 610-618.

Lorenzo JM, Pateiro M, Franco D (2013). Influence of muscle type on physicochemical and sensory properties of foal meat. Meat Science 94 (1): 77-83.

Lorenzo JM, Crecente S, Franco D, Sarriés MV, Gómez M (2014). The effect of livestock production system and concentrate level on carcass traits and meat quality of foals slaughtered at 18 months of age. Animal 8 (3): 494-503.

Polidori P, Pucciarelli S, Ariani A, Polzonetti V, Vincenzetti S (2015). A comparison of the carcass and meat quality of Martina Franca donkey foals aged 8 or 12 months. Meat Science 106: 6-10.

Rentfrow G, Linville ML, Stahl CA, Olson KC, Berg EP (2004). The effects of the antioxidant lipoic acid on beef Longissimus bloom time. Journal of Animal Science 82: 3034-3037.

Roncalés $P$ (2001). Transformación del músculo en carne: rigor mortis y maduración. En: Enciclopedia de la Carne y de los Productos Cárnicos 1 (Ed. Martín y Macías) pp. 291-312. Cáceres, España.

Ruiz M, Insausti K, Beriain MJ, Lorenzo JM, Sarriés MV (2016). Description of the training methodology employed to describe foal meat attributes. Book of abstracts of the 7th European Conference on Sensory and Consumer Research Biennial Meeting of the Eurosense, 11-14 de Septiembre, Dijon, Francia, P154.

Sañudo C (1993). La calidad organoléptica de la carne (IV). Mundo Ganadero 6, 68-73.

Sarriés MV, Beriain MJ (2005). Carcass characteristics and meat quality of male and female foals. Meat Science 70: 141-152.

Sarriés MV, Beriain MJ (2006). Color and texture characteristics in meat of male and female foals. Meat Science 74: 738-745.

Sarriés MV, Murray BE, Troy D, Beriain MJ (2006). Intramuscular and subcutaneous lipid fatty acid profile composition in male and female foals. Meat Science 72 (3): 475-485. 
Seydim AC, Acton JC, Hall MA, Dawson PL (2006). Effects of packaging atmospheres on shelf-life quality of ground ostrich meat. Meat Science 73 (3): 503-510.

Spanier AM (1992). Current approaches to the Study of meat flavor quality. En: Food Science y Human Nutrition (Ed. G. Charalambous) pp 695-709 Elsevier Science Publishers BV.

Tarladgis BG, Watts BM, Yonathan M (1960). A distillation method for the quantitative determination of malonaldehyde in rancid foods. Journal of the American Oil Chemists' Society 37 (1): 44-48.
Tateo A, De Palo P, Ceci E, Centoducati P (2008). Physicochemical properties of meat of Italian Heavy Draft horses slaughtered at the age of eleven months. Journal of Animal Science 86: 1205-1214.

Zakrys PI, Hogan SA, O'Sullivan MG, Allen P, Kerry JP (2008). Effects of oxygen concentration on the sensory evaluation and quality indicators of beef muscle packed under modified atmosphere. Meat Science 79 (4): 648-655.

(Aceptado para publicación el 29 de noviembre de 2017) 